\title{
Presigmoid Approach to Vertebrobasilar Artery Aneurysms : A Series of 31 Patients and Review of the Literature
}

\section{Tjahjadi, Mardjono}

2016-08

Tjahjadi , M , Niemelä , M , Kivelev , J Serrone , J , Maekawa , H , Jahromi , B R , Kerro , O , Hafez , A , Lehto , H , Kivisaari , R \& Hernesniemi , J 2016 , ' Presigmoid Approach to Vertebrobasilar Artery Aneurysms : A Series of 31 Patients and Review of the Literature ', World Neurosurgery , vol. 92 , pp. 313-322 . https://doi.org/10.1016/j.wneu.2016.05.001

http://hdl.handle.net/10138/224032

https://doi.org/10.1016/j.wneu.2016.05.001

publishedVersion

Downloaded from Helda, University of Helsinki institutional repository.

This is an electronic reprint of the original article.

This reprint may differ from the original in pagination and typographic detail.

Please cite the original version. 


\section{Presigmoid Approach to Vertebrobasilar Artery Aneurysms: A Series of 31 Patients and Review of the Literature \\ Mardjono Tjahjadi, Mika Niemelä, Juri Kivelev, Joseph Serrone, Hidetsugu Maekawa, Behnam Rezai Jahromi, Oleg Kerro, Ahmad Hafez, Hanna Lehto, Riku Kivisaari, Juha Hernesniemi}

OBJECTIVE: The presigmoid approach can be used to treat vertebrobasilar artery aneurysms when circumstances require more operative exposure. High morbidity and mortality in these cases have been reported. In this study, we describe our modified presigmoid approach for vertebrobasilar artery aneurysms and our clinical results.

METHODS: This series includes patients with vertebrobasilar aneurysms clipped via the modified presigmoid approach at the Department of Neurosurgery at Helsinki University Hospital from 1998 to 2014. Data were collected from the operating record books, patients' medical records, and a radiologic database server.

RESULTS: Thirty-three presigmoid procedures of 31 patients were performed to treat 34 aneurysms (14 ruptured, 20 unruptured). The aneurysms had a mean distance from the posterior clinoid process to the aneurysm neck of 12.2 $\mathrm{mm}$ (range, 0-26.6 mm). A favorable outcome was achieved in 21 patients (64\%). A favorable outcome was achieved in $74 \%$ of unruptured and good-grade patients, whereas favorable outcome was achieved in only $36 \%$ of poor-grade patients. Complete or near-complete occlusion was achieved in $\mathbf{7 9 \%}$. Larger aneurysms, fusiform morphology, and anterior dome projection had lower occlusion rates.

CONCLUSIONS: We have described our experiences of using the presigmoid approach to treat vertebrobasilar aneurysms. The clinical and radiographic results are acceptable given the complex location and configuration of the treated aneurysms. Unfavorable outcomes are related to the poor admission Hunt and Hess grade, aneurysm morphology, and aneurysm size.

\section{INTRODUCTION}

he presigmoid approach (also referred to as the posterior transpetrosal approach or combined suprainfratentorial approach) is considered one of the most complex procedures in microneurosurgery. This approach was originally introduced as a surgical route to treat the ventral brainstem and clival tumors but it has also been applied to posterior circulation aneurysms that were not feasible for standard pterional, subtemporal, or retrosigmoid approaches. ${ }^{\mathrm{I}}$ In the early years of skullbase surgery, this procedure was performed through the infratentorial corridor, sacrificing the sigmoid sinus and labyrinth. This approach resulted in hearing loss and complications related to venous hypertension. ${ }^{2,3}$ Hakuba et al. ${ }^{4,5}$ later introduced a modification of a combined partial labyrinthectomy and petrous apicectomy to treat retrochiasmatic craniopharyngiomas and clival meningiomas. This technique avoided sacrifice of the sigmoid sinus with improved hearing outcomes and fewer complications related to venous insufficiency.

Recently, many less invasive variations of the presigmoid approach have been described, including retrolabyrinthine, transcrusal, minimum combined transpetrosal, and partial superoposterior petrosectomy. ${ }^{6-12}$ However, the presigmoid approach still carries a relatively high morbidity $(62 \%)$ and

\section{Key words \\ - Angioplasty \\ - Aortic arch reconstruction \\ - Arterial occlusive disease \\ - Intracranial stenting \\ - Mechanical thrombolysis \\ - Stroke}

\section{Abbreviations and Acronyms \\ BA: Basilar artery \\ CSF: Cerebrospinal fluid \\ GOS: Glasgow Outcome Score \\ PCP: Posterior clinoid process \\ SPS: Superior petrosal sinus}

Department of Neurosurgery, University of Helsinki and Helsinki University Hospital, Helsinki, Finland

To whom correspondence should be addressed: Mardjono Tjahjadi, M.D.

[E-mail: joy_mardjono@yahoo.com]

Citation: World Neurosurg. (2016) 92:313-322.

http://dx.doi.org/10.1016/j.wneu.2016.05.001

Journal homepage: www.WORLDNEUROSURGERY.org

Available online: www.sciencedirect.com

1878-8750/\$ - see front matter (c) 2016 Elsevier Inc. All rights reserved. 
mortality $(9 \%) .{ }^{\text {IO,II }}$ After the publication of the ISAT (International Subarachnoid Aneurysm Trial) and ISUIA (International Study of Unruptured Intracranial Aneurysms) trials reporting competitive results of endovascular therapy for aneurysm treatment, endovascular therapy has become more common in general and especially for treating posterior circulation aneurysms. ${ }^{\mathrm{I}-\mathrm{I} 5}$ Nonetheless, not all vertebrobasilar aneurysms can be treated well by endovascular means. Some drawbacks of the endovascular treatment of cerebral aneurysms in either clinical or radiologic evaluation have been reported. ${ }^{16-19}$ In certain cases, microsurgery still has a role in treating posterior circulation aneurysms.

In this study, we review our experience in using a modified presigmoid approach to treat vertebrobasilar aneurysms and to correlate the preoperative clinical and radiologic characteristics to patient outcomes.

\section{METHODS}

This retrospective review includes a consecutive series of patients with vertebrobasilar aneurysm treated by our modified presigmoid approach at the Department of Neurosurgery at Helsinki University Central Hospital (catchment area of I. 8 million inhabitants) between 1998 and 2014. Data were collected from the operating record books, patients' medical records, and a radiologic database server.

\section{Patient Data}

Our review included 3492 patients with 4677 intracranial aneurysms. From this population, 6or aneurysms were located in the vertebrobasilar system. A microsurgical procedure was performed for 42I aneurysms, endovascular therapy in 66 aneurysms, and conservative management in II4. The cases managed conservatively were mainly because of poor clinical condition at presentation for ruptured cases or small aneurysm size $(<3 \mathrm{~mm})$ in unruptured cases indicating a lower risk for rupture.

\section{Clinical Data}

Preoperative clinical condition was assessed using the Hunt and Hess score. ${ }^{20}$ The outcome after surgery was assessed using the modified Rankin Scale with classification into 3 categories: favorable if the condition is similar to the preoperative condition or with minor neurologic signs that did not disturb their life activities including walking satisfactorily (Glasgow Outcome Score [GOS] 4-5); unfavorable if the condition is worse postoperatively with moderate to severe neurologic deficit (GOS $2-3$ ); and death (GOS I).

\section{Radiologic Measurement}

Radiologic data for the study were obtained from the available computed tomography angiography and digital subtraction angiography images. Radiologic images were analyzed both in 2 and 3 dimensions using the GE Centricity RA 6oo software (GE Medical Systems, Milwaukee, Wisconsin, USA) and the IMPAX software version 5.3 (Agfa, Mortsel, Belgium). The aneurysm characteristics for statistical analysis included the size of aneurysm dome and neck, vertical distance from the aneurysm neck to the posterior clinoid process (PCP), the lateralization of the aneurysm neck or parent artery with respect to the clivus, direction of aneurysm dome projection, and postoperative angiographic occlusion of the aneurysm.
Patients with incomplete radiologic data were excluded from this study. Aneurysm size was categorized as: very small, $<3 \mathrm{~mm}$; small, 3-6 mm; medium, 7-I3 mm; large, $\mathrm{I} 4-24 \mathrm{~mm}$; or giant, $\geq 25 \mathrm{~mm}$. The radiologic evaluation of aneurysm occlusion was performed on the first postoperative day and divided into 3 categories: complete occlusion, near-complete occlusion (any signs of neck remnant), and incomplete occlusion (fundus remnant).

\section{Operative Technique and Methods}

Although the details of the presigmoid approach have been described elsewhere, below we review our modifications of this approach (Figure 1). ${ }^{2 \mathrm{I}}$

Patient Position and Cerebrospinal Fluid Drainage. The patient is placed in the park bench position with the mastoid process as the highest point. The upper shoulder is carefully retracted caudally and backward with tape to increase the surgeon's working space. Lumbar cerebrospinal fluid (CSF) drainage (50 $\mathrm{mL}$ ) helps to provide a slack brain before the dural opening and this action facilitates the subtemporal route to the interpeduncular cistern.

Skin Incision And Craniotomy. The I-layer horseshoe incision forming musculocutaneous flap is performed from a point about I $\mathrm{cm}$ anterior and superior of the root of the zygoma, arching posterosuperiorly $2-3 \mathrm{~cm}$ over the ear and stopping $2 \mathrm{~cm}$ posterior to the occipitomastoid suture. This myocutaneous flap is retracted frontocaudally and fixated with several Sugita spring-hook retractors to expose the temporal and occipital-retrosigmoid area. The craniotomy is made to expose the dura of the middle fossa and lateral suboccipital area. An extradural dissection is performed to expose the subtemporal dural and the upper part of the sinodural angle.

Temporal Bone Drilling. Drilling is performed under high magnification of the operating microscope. The base of the squamous part of the temporal bone is drilled first to support an adequate supratentorial surgical corridor with minimal retraction of the temporal lobe. Drilling the posterosuperior mastoid part of the temporal bone increases the exposure of the sinodural angle, which has been exposed after the craniotomy. This angle or the presigmoid dura is exposed as necessary and drilling stops at the level of the anthrum without compromising the structures of the middle or inner ear. During this procedure, gradual and cautious detachment of the dura mater from the inner surface of the petrous-mastoid temporal bone is important to prevent damage to the sigmoid sinus. The transverse and sigmoid sinuses are left intact. The postoperative result of the temporal bone drilling can be seen in Figure 2.

Dural Opening and Cutting of the Tentorium. The dura mater is opened in a J shape that involves the subtemporal and presigmoid areas. The first opening is a horizontal incision of the subtemporal dura mater that extends posteriorly toward the sinodural angle. The second opening is a vertical incision of the presigmoid dura mater. These 2 dural openings are connected by dividing the superior petrosal sinus (SPS). The tentorium is cut to the incisura just posterior to the insertion of the fourth nerve into the tentorial edge. The mobilized anterior flap of the tentorium is folded anteriorly and fixed to the anterior tentorium with small aneurysm clips (Figure 1). 

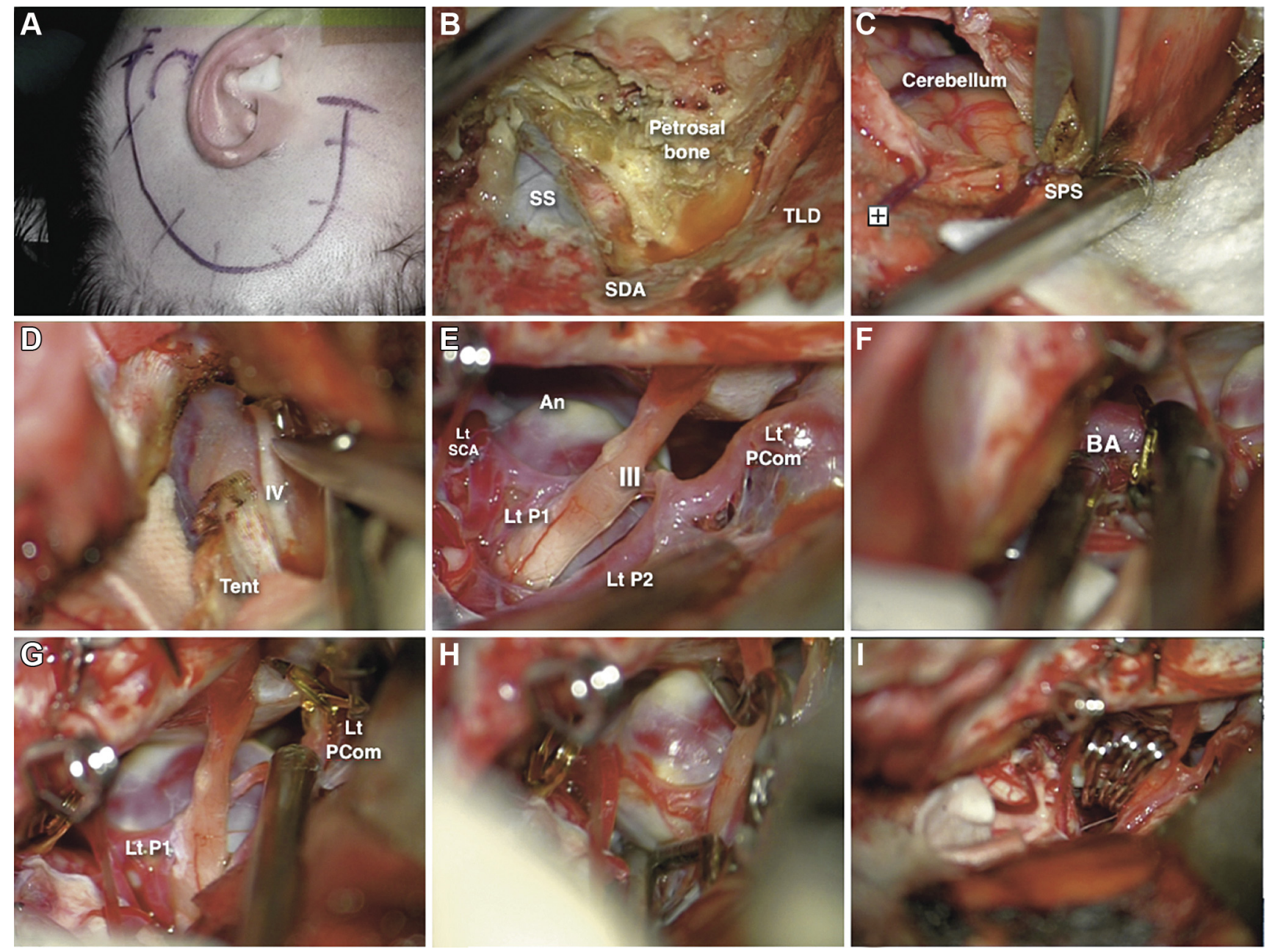

Figure 1. Steps of the presigmoid approach to clip a basilar tip aneurysm. (A)

artery (BA). (G) Distal temporary clip of left posterior communicating artery (Lt PCom). (H) Neck clip application. (I) Definitive tandem clip application. III, Skin incision marking. (B) The exposure after the craniotomy and temporal bone drilling. (C) Opening the dura mater and cutting the superior petrosal sinus (SPS). (D) The tentorium (Tent) is cut toward its edge with

preservation of the fourth nerve. (E) Aneurysm exposure with its surrounding neurovascular anatomy. (F) Proximal temporary clip of basilar oculomotor nerve; IV, trochlear nerve; An, aneurysm; Lt P1, left posterior cerebral artery segment 1; Lt P2: left posterior cerebral artery segment 2; Lt SCA, left superior cerebellar artery; SDA, sinodural angle: SPS, superior petrosal sinus; SS, sigmoid sinus; TLD, temporal lobe dura.

Closing. The closure for this approach is of the utmost importance to avoid postoperative CSF leakage and infection. The dura mater is closed in a watertight fashion and if necessary reinforced by dural sealants. Any open mastoid air cells are identified and sealed with a pedicle of the temporal muscle flap. This flap promotes the granulation between the muscle and the air-cell mucosa that closes the defect. Bone, soft tissue, and skin closure should be performed in layers under high magnification of the operating microscope.

\section{RESULTS}

From 1998 to 20I4, the senior author (J.H.) performed 36 presigmoid approaches to operate on 37 vertebrobasilar aneurysms in 34 patients. Three patients were excluded because of incomplete radiologic data for the analysis, leaving 3I patients with 33 procedures on 34 vertebrobasilar aneurysms. Two of the patients came from outside Finland but could be followed up postoperatively.

General Characteristics of the Patients

The male/female ratio was I:I (I6 men and 17 women) with a mean age of 47.9 years (range, 28-68 years). Three of 3 I patients had 2 basilar artery aneurysms. Two of these patients required bilateral approaches because of the different origin and projections of the basilar artery aneurysms. Twenty aneurysms $(59 \%)$ were ruptured with a median Hunt and Hess score of 3 (Table 1). The mean duration of surgery from skin incision to skin closure was I89 minutes (range, $70-257$ minutes). 


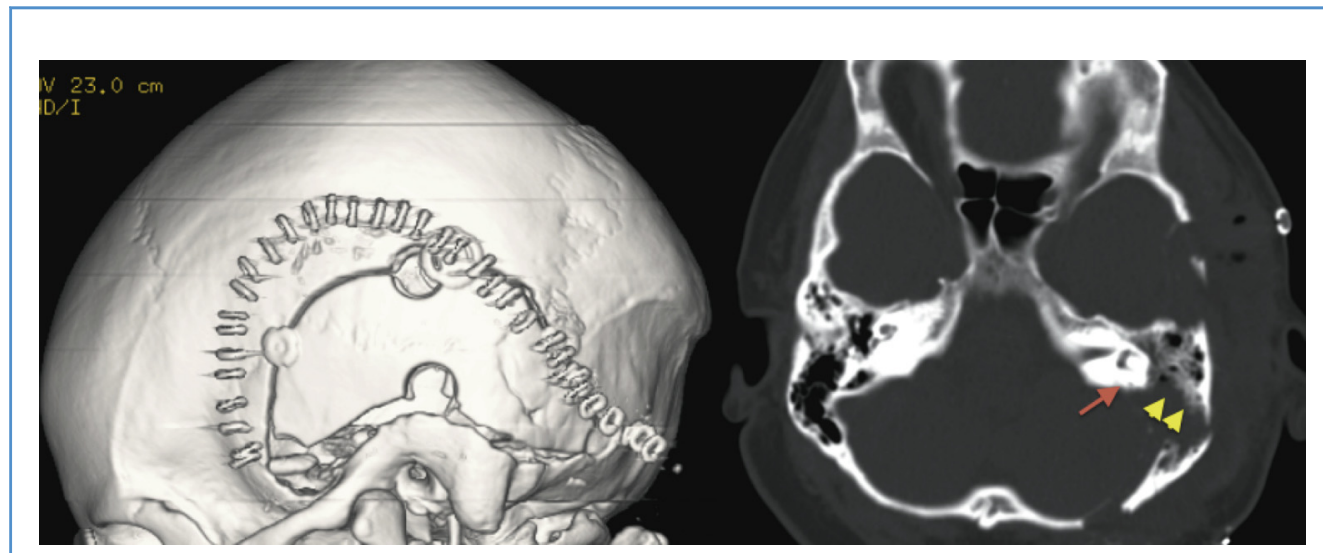

Figure 2. The postoperative computed tomographic bone reconstruction showed the area of craniotomy and temporal bone drilling. The minimal bone drilling allows the surgery to be faster and safer (yellow arrows). Red arrow, the inner ear structures that were left intact.

\section{General Aneurysm Characteristics}

Aneurysm Morphology. The aneurysms were saccular in 29 cases $(85 \%)$, fusiform in 4 cases (12\%), and dissecting in I case $(3 \%)$. The mean aneurysm dome size was $9.8 \mathrm{~mm}$ (range, $2.6-38.4 \mathrm{~mm}$ ).

Table 1. Clinical Characteristics of 33 Procedures Using the Presigmoid Approach in Our Series

\begin{tabular}{|l|c|}
\hline Description & Value \\
\hline Age (years), mean (range) & $47.9(28-68)$ \\
\hline Sex & $16(49)$ \\
\hline Male & $17(51)$ \\
\hline Female & \\
\hline Preoperative Hunt and Hess grade & $14(43)$ \\
\hline 0 & $6(18)$ \\
\hline 1 & $3(9)$ \\
\hline 2 & $6(18)$ \\
\hline 3 & $3(9)$ \\
\hline 4 & $1(3)$ \\
\hline 5 & $189(70-257)$ \\
\hline Operation duration (skin to skin) (minutes), mean (range) & \\
\hline Approach side & $24(73)$ \\
\hline Right side & $9(27)$ \\
\hline Left side & $21(64)$ \\
\hline Postoperative outcome & $7(21)$ \\
\hline Favorable & $5(15)$ \\
\hline Unfavorable & \\
\hline Dead & \\
\hline Values are number (\%) unless otherwise indicated. & \\
\hline
\end{tabular}

The mean aneurysm neck size for saccular and dissecting aneurysms was $5.5 \mathrm{~mm}$, with a mean dome/neck ratio of I.6. Most aneurysms were small and medium-sized, accounting for $\mathrm{I} 7(35 \%)$ and $\mathrm{Io}(29 \%)$ cases, respectively (Table 1).

Aneurysm Location. The most common site of the aneurysm was the basilar trunk, accounting for $\mathrm{I} 4$ aneurysms $(4 \mathrm{I} \%)$. In decreasing order of frequency, the remaining aneurysms were located at the basilar bifurcation, basilar-superior cerebellar artery, basilar-anterior inferior cerebellar artery, vertebrobasilar junction, and distal vertebral artery (Table 2 and Figure 3). The mean distance from the PCP to the proximal neck of the aneurysm was $12.2 \mathrm{~mm}$ (range, $0-26.6 \mathrm{~mm}$ ). The lateralization of the aneurysm proximal neck was as follows: 26 midline aneurysms (76\%), 5 right sided ( $15 \%)$, and 3 left sided (9\%). The presigmoid approach was performed from the right side in 24 procedures $(73 \%)$ and from the left side in 9 procedures $(27 \%)$. Aneurysms at the midline were approached from the right side in $2 \mathrm{I}$ patients $(8 \mathrm{I} \%)$ and from the left side in 5 patients $(\mathrm{I} 9 \%)$.

\section{Radiographic Outcome}

A complete or near-complete aneurysm occlusion was achieved in 27 cases $(79 \%)$. The mean aneurysm size for aneurysms with complete occlusion, neck remnant, and fundus remnant was 7.8 $\mathrm{mm}, 9.9 \mathrm{~mm}$, and $\mathrm{I} 6.7 \mathrm{~mm}$, respectively. Several other factors were also found to predict fundus remnants including fusiform morphology $(75 \%)$, vertebrobasilar junction location $(50 \%)$, and anterior dome projection (50\%) (Table 3 and Figure 4).

\section{Clinical Outcome}

Clinical outcome was evaluated with a mean follow-up of 6.2 months (range, $\mathrm{I}-30$ months). The median GOS in this series was 4. Postoperative CSF leak was seen in 2 cases $(6 \%)$; one of them also developed a postoperative wound infection. The CSF leaks were transient and healed with spinal drainage and antibiotics. A favorable outcome was achieved in 2I patients $(64 \%)$. A favorable outcome was more common in unruptured cases $(7 \mathrm{I} \%)$ compared 
Table 2. Patient's General Outcome According to the Clinical and Radiologic Factors

\begin{tabular}{|c|c|c|c|c|}
\hline Description & Favorable & Unfavorable & Dead & Total \\
\hline Age (years), mean (range) $(\mathrm{n}=33$ ) & $47.5(30-68)$ & $47.6(28-62)$ & $50(36-65)$ & \\
\hline \multicolumn{5}{|l|}{ Hunt and Hess score $(n=33)$} \\
\hline Good grade $(0-2)$ & $17(74)$ & $5(22)$ & $1(4)$ & $23(68)$ \\
\hline Poor grade $(3-5)$ & $4(36)$ & $2(28)$ & $4(36)$ & $10(32)$ \\
\hline \multicolumn{5}{|l|}{ Aneurysm morphology ( $n=34$ ) } \\
\hline Saccular & $19(66)$ & $7(24)$ & $3(10)$ & $29(85)$ \\
\hline Fusiform & $2(50)$ & $1(25)$ & $1(25)$ & $4(12)$ \\
\hline Dissecting & $0(0)$ & $0(0)$ & $1(100)$ & 1 (3) \\
\hline Neck size $(\mathrm{mm})$, mean (range) $(n=30)$ & $4.3(1.7-12.8)$ & $8.2(2.4-20.4)$ & $5.4(4.8-6.1)$ & \\
\hline Dome size $(\mathrm{mm})$, mean (range) $(\mathrm{n}=34)$ & $8.9(2.6-38.4)$ & $14.8(5.5-28.6)$ & $5.8(2.6-7.3)$ & \\
\hline Very small (<3 mm) & $4(80)$ & $0(0)$ & $1(20)$ & $5(15)$ \\
\hline Small $(3-7 \mathrm{~mm})$ & $9(75)$ & 1 (8) & $2(17)$ & $12(35)$ \\
\hline Medium (7-14 mm) & $5(50)$ & $3(30)$ & $2(20)$ & $10(29)$ \\
\hline Large (14-24 mm) & $2(50)$ & $2(50)$ & $0(0)$ & $4(12)$ \\
\hline Giant (>24 mm) & $1(33)$ & $2(67)$ & & 3 (9) \\
\hline Distance from the tip of posterior clinoid process to the proximal aneurysm distance & $13(0-26.6)$ & $12(5.6-26.4)$ & $8.6(4.4-15.1)$ & \\
\hline \multicolumn{5}{|l|}{ Aneurysm origin $(n=34)$} \\
\hline Basilar bifurcation & $6(55)$ & $3(27)$ & $2(18)$ & $11(32)$ \\
\hline Basilar-superior cerebellar artery & $2(50)$ & $2(50)$ & $0(0)$ & $4(12)$ \\
\hline Basilar trunk & $9(64)$ & $2(14)$ & $3(21)$ & $14(41)$ \\
\hline Basilar-anterior inferior cerebellar artery & $2(100)$ & $0(0)$ & $0(0)$ & $2(6)$ \\
\hline Vertebrobasilar junction & $1(50)$ & $1(50)$ & $0(0)$ & $2(6)$ \\
\hline Distal vertebral & $1(100)$ & $0(0)$ & $0(0)$ & 1 (3) \\
\hline \multicolumn{5}{|l|}{ Dome projection $(\mathrm{n}=34)$} \\
\hline Superior & $3(43)$ & $2(29)$ & $2(29)$ & $7(20)$ \\
\hline Posterior & $4(57)$ & $3(43)$ & $0(0)$ & $7(20)$ \\
\hline Anterior & $3(50)$ & $2(33)$ & $1(17)$ & $6(18)$ \\
\hline Right & $7(70)$ & $1(10)$ & $2(20)$ & $10(30)$ \\
\hline Left & $4(100)$ & $0(0)$ & $0(0)$ & $4(12)$ \\
\hline
\end{tabular}

with ruptured cases (58\%). An unfavorable outcome occurred in 7 patients $(36 \%)$ and 5 patients died (15\%) (Table 2). Four of the mortalities $(80 \%)$ had poor Hunt and Hess grade on admission. The only unruptured case that had a fatal outcome was a patient with Ehlers-Danlos syndrome with a medium-sized basilar trunk aneurysm. This patient experienced an intraoperative rupture because the arterial wall was fragile. The situation was successfully managed, Indocyanine green angiography showed well-contrasted filling of the basilar artery and perforators, but severe basilar artery vasoconstriction occurred postoperatively, with subsequent postoperative brainstem stroke resulting in death.

Postoperative permanent cranial nerve deficit was seen in 5 patients (I6\%) (Table 4). Three patients developed cranial nerve
VII and VIII palsy, I patient developed an abducens palsy, and I patient developed cranial nerve III and IV palsy. Transient cranial nerve deficit occurred in 13 patients $(39 \%)$. Six of them (46\%) had multiple cranial nerve deficits. Diplopia associated with abducens nerve palsy was the most common transient postoperative cranial nerve deficit and was seen in ro patients $(32 \%)$. Hemiparesis occurred in 8 patients, with $7(88 \%)$ patients recovering to normal motor function.

\section{DISCUSSION}

The presigmoid approach for treating vertebrobasilar aneurysms is used in a selective manner. ${ }^{33,34} \mathrm{~A}$ current literature review of the 


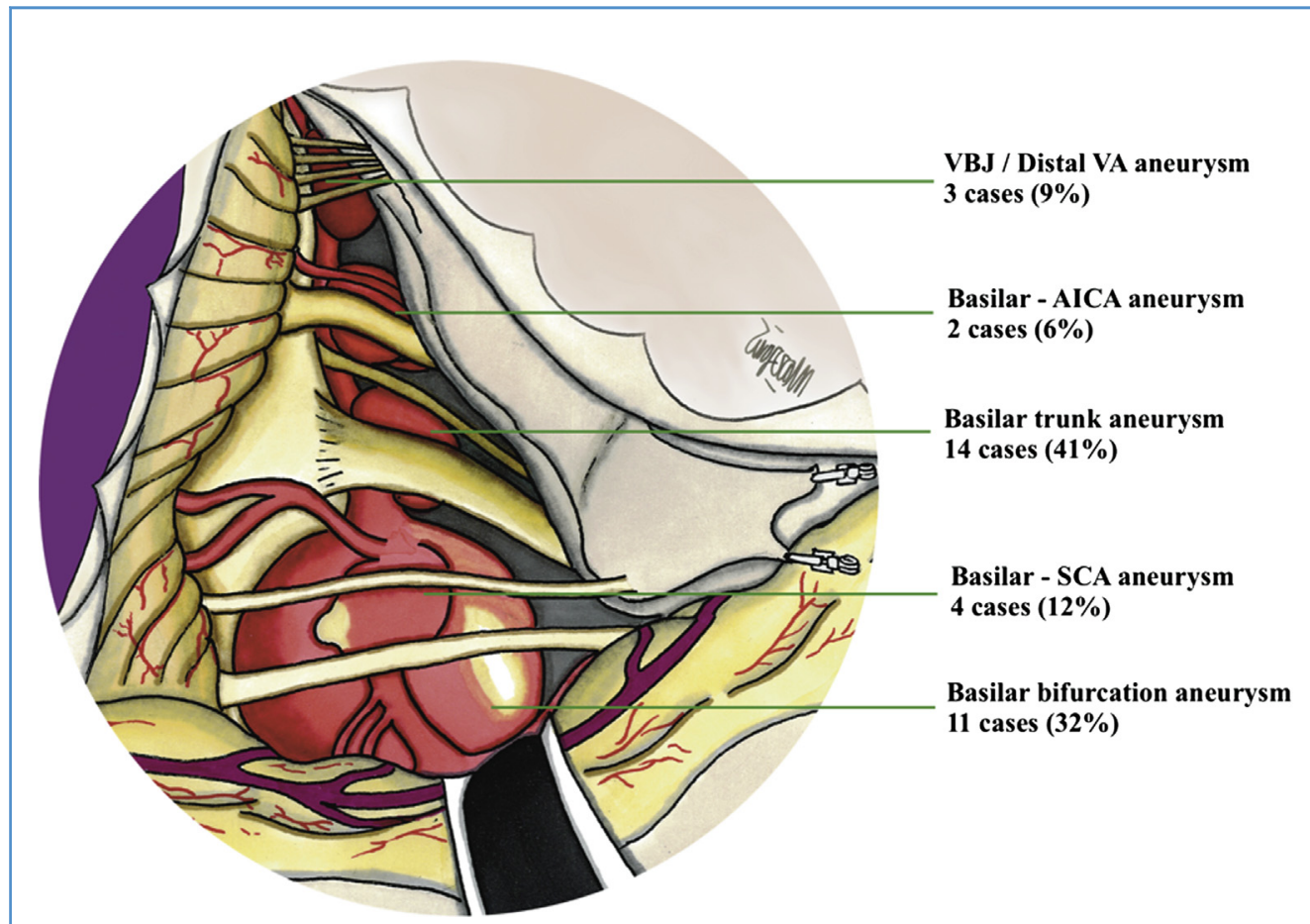

Figure 3. The location of basilar artery aneurysms that were clipped using our modified presigmoid approach. AICA, anterior inferior cerebellar artery; SCA, superior cerebellar artery; VA, vertebral artery; VBJ, vertebrobasilar junction.

posterior transpetrosal approach to aneurysms in the posterior circulation found reports of only 34 patients published in the last 20 years. $^{\text {Io }}$ Our series comprises 33 procedures in $3 \mathrm{I}$ patients, making it the largest series to date; moreover, all the consecutive cases were treated by a single surgeon (J.H.) in a single center. No patient was lost to follow-up.

In the present series, we showed a simplified classic posterior petrosal/presigmoid approach with a tailored petrosal bone drilling. This approach modification was invented by the senior author (J.H.). In most cases, we do not need to drill the petrosal bone extensively and usually stop before reaching the semicircular canals. For this more limited bony removal to still provide the necessary exposure, cooperation with the neuroanesthetist to maintain a slack brain and the use of very high magnification during surgery are required..$^{35}$ With this strategy, surgical times are limited to approximately 3 hours. Only 2 patients $(6 \%)$ developed postoperative CSF leakage, which was treated without surgical intervention. These findings are interesting because the literature reports a CSF leak rate of $21 \% .{ }^{\text {IO }}$ We believe the technique of a tailored bone drilling and closing the opened air cells with a temporalis muscle pedicle are keys to the prevention for CSF leakage.

Until now, many surgical approaches have been proposed for reaching retroclival basilar artery aneurysms, including anterolateral, lateral, or posterolateral routes. ${ }^{6,33,36,37}$ The anterolateral route (eg, pterional, orbitozygomatic, pretemporal, and their modifications) gives a relatively shorter working distance to reach the region..$^{38}$ The main disadvantage of this approach is the limited access to the lower segment of the basilar artery, even after the removal of the PCP and petrous apex..$^{39^{-42}}$ The lateral route consists of a subtemporal approach and its extensions. ${ }^{43-46}$ This approach for basilar aneurysms was introduced and popularized by Charles Drake and continues to be used regularly by the senior author (J.H.). ${ }^{44,47}$ Additional removal of the petrosal apex, which was popularized by Kawase et al., ${ }^{45}$ allows surgeons to reach basilar aneurysms up to $18 \mathrm{~mm}$ below the sellar floor. ${ }^{48}$

The posterolateral route (eg, presigmoid, retrosigmoid, and their modifications) gives a relatively longer working distance, but it exposes lesions at almost all segments of the basilar artery. The presigmoid approach with opening of the dura over the petrous temporal bone, subtemporal dura, and sectioning of the tentorium creates a supratentorial and infratentorial corridor for exposure of the vertebrobasilar system. ${ }^{\mathrm{I}, 3,4, \mathrm{I} 2}$ The disadvantage of the presigmoid approach is its complexity, resulting in a longer operative duration and higher risk of injuring venous structures, cranial nerves, and the vestibulocochlear system. In some specific situations in which the patient has a petrosal or sphenopetrosal venous system, sacrifice of the SPS and cutting the tentorium are not safe. Hafez et al. ${ }^{49}$ recommended freeing the SPS from its groove to the Meckel cave and cutting the tentorium below the SPS. In our experience, if the patient has such a venous system group, the senior author (J.H.) works from the supratentorial and 
Table 3. The Aneurysm Occlusion Rate Characteristics

\begin{tabular}{|c|c|c|c|}
\hline Description & Complete (number) (\%) & Near-Complete (number) (\%) & Incomplete (number) (\%) \\
\hline \multicolumn{4}{|l|}{ Aneurysm presentation } \\
\hline Unruptured & $6(43)$ & $3(21)$ & $5(36)$ \\
\hline Ruptured & $17(85)$ & $1(5)$ & $2(10)$ \\
\hline \multicolumn{4}{|l|}{ Aneurysm morphology } \\
\hline Saccular & $21(72)$ & $4(14)$ & $4(14)$ \\
\hline Fusiform & $1(25)$ & $0(0)$ & $3(75)$ \\
\hline Dissecting & $1(100)$ & $0(0)$ & $0(0)$ \\
\hline Posterior clinoid process to aneurysm distance & $11.4(0-26.6)$ & $12.9(8.2-16.8)$ & $15.4(0-26.4)$ \\
\hline Neck size $(\mathrm{mm})$, mean (range) ${ }^{*}$ & $5.0(1.7-20.4)$ & $7.1(4.7-12.8)$ & $5.9(2.6-9.5)$ \\
\hline \multicolumn{4}{|l|}{ Aneurysm origin } \\
\hline Basilar bifurcation & $7(64)$ & $2(18)$ & $2(18)$ \\
\hline Basilar-superior cerebellar artery & $4(100)$ & $0(0)$ & $0(0)$ \\
\hline Basilar trunk & $8(57)$ & $2(14)$ & $4(29)$ \\
\hline Basilar-anterior inferior cerebellar artery & $2(100)$ & $0(0)$ & $0(0)$ \\
\hline Vertebrobasilar junction & $1(50)$ & $0(0)$ & $1(50)$ \\
\hline Distal vertebral & $1(100)$ & $0(0)$ & $0(0)$ \\
\hline Dome size $(\mathrm{mm})$, mean (range) & $7.8(2.6-28.6)$ & $9.9(7-13.4)$ & $16.7(6.4-38.4)$ \\
\hline Very small $(<3 \mathrm{~mm})$ & $5(100)$ & $0(0)$ & $0(0)$ \\
\hline Small (3-7 mm) & $11(92)$ & $0(0)$ & $1(8)$ \\
\hline Medium (7-14 mm) & $4(40)$ & $4(40)$ & $2(20)$ \\
\hline Large (14-24 mm) & $2(50)$ & $0(0)$ & $2(50)$ \\
\hline Giant (>24 mm) & $1(33)$ & $0(0)$ & $2(67)$ \\
\hline \multicolumn{4}{|l|}{ Dome projection } \\
\hline Superior & $6(86)$ & $0(0)$ & $1(14)$ \\
\hline Posterior & $4(57)$ & $1(14)$ & $2(28)$ \\
\hline Anterior & $3(50)$ & $0(0)$ & $3(50)$ \\
\hline Right & $7(70)$ & $2(20)$ & $1(10)$ \\
\hline Left & $3(75)$ & $1(25)$ & $0(0)$ \\
\hline
\end{tabular}

infratentorial corridor, cutting only the edge of the tentorium to increase the exposure.

Previous literature emphasizes the level of the aneurysm neck relative to the bony structures (such as the PCP or floor of the sellae turcica) for selection of surgical approaches..$^{37,48}$ Aziz et al. ${ }^{48}$ proposed using the presigmoid approach if the aneurysm is located more than $18 \mathrm{~mm}$ below the floor of the sellae turcica. In the present series, the aneurysms treated with a presigmoid approach had an average distance, being $12.2 \mathrm{~mm}$ below the PCP, placing most aneurysms well above the cutoff point proposed by Aziz et al. We prefer to use the distance of aneurysms from the PCP to determine surgical approaches because this structure is encountered in surgery, whereas the floor of the sellae turcica is only radiographically identified.

Surgical Outcomes and Complications

Even when taking into account the unselected nature of the series, the results are comparable with the existing literature (Table 4). ${ }^{\text {IO }}$ The incidence of permanent postoperative cranial nerve injury and hemiparesis occurred in $16 \%$ and $3.2 \%$, respectively. The death rates were highly related to the poor Hunt and Hess scale on admission. ${ }^{\text {IO }}$ In addition, we believe that the learning curve led to for lower morbidity at the end of the series. 


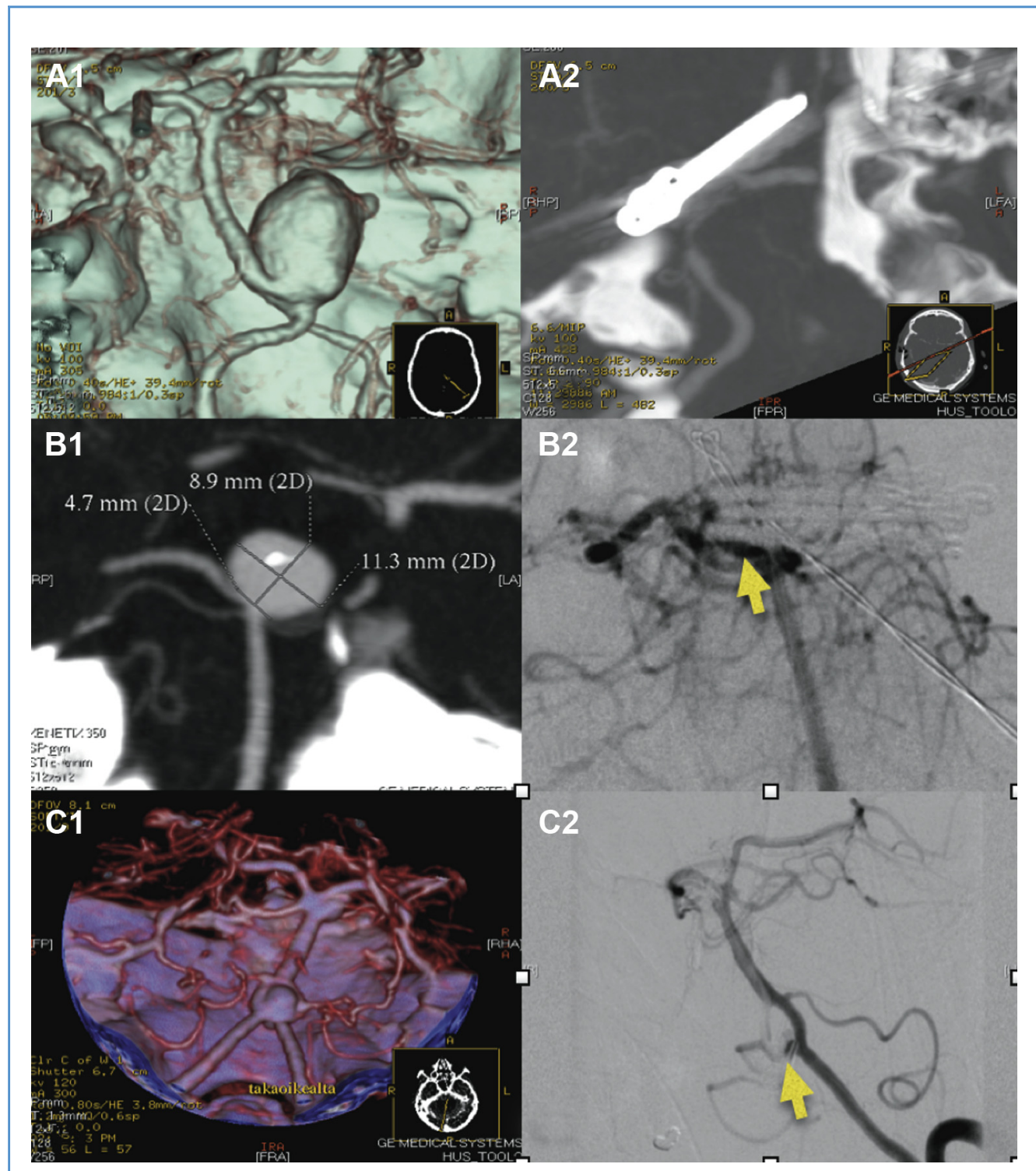

Figure 4. (A1, A2) Pre- and postoperative result of complete clipping of a large proximal basilar trunk aneurysm.

(B1, B2) Pre- and postoperative result of the neck residual of the medium-sized basilar tip aneurysm; some part of the neck remnant is still seen on the postoperative angiogram (yellow arrow). (C1, C2). Pre- and postoperative image of both basilar-superior cerebellar artery and fenestrated vertebrobasilar junction aneurysm. The basilar-superior cerebellar artery aneurysm is completely clipped, but there is a small neck and fundus remnant of the fenestrated vertebrobasilar junction aneurysm (yellow arrow).

Clip occlusion rates of the aneurysms were evaluated by postoperative computed tomography angiography or digital subtraction angiography. For saccular aneurysms, the rate for complete occlusion was $7 \mathrm{I} \%$ (nontitanium clips cause artifact, making full estimation of neck remnants challenging). This finding is consistent with the finding of Kivisaari et al. in $2004,^{50}$ who reported a $74 \%$ rate of occlusion for vertebrobasilar saccular aneurysms operated by the senior author (J.H.). These investigators also considered the aneurysm location, small gap, and size of the aneurysms as reasons for the postclipping aneurysm remnants, which were consistent with our findings.

Endovascular and Microsurgery Perspective

With endovascular therapy using natural routes, one can avoid extensive skull-base approaches and cranial nerve deficits, which 
Table 4. The Multiauthor Review of Presigmoid Approach

Experience for Vertebrobasilar Aneurysm (Adapted from Gross et al. $^{10}$ )

\begin{tabular}{|c|c|c|c|c|}
\hline Reference & $\begin{array}{l}\text { Number } \\
\text { of Cases }\end{array}$ & $\begin{array}{c}\text { Cerebrospinal } \\
\text { Fluid Leak, } \\
\text { n (\%) }\end{array}$ & $\begin{array}{c}\text { Permanent } \\
\text { Cranial } \\
\text { Nerve Deficit, } \\
\text { n (\%) }\end{array}$ & Mortality \\
\hline $\begin{array}{l}\text { Solomon et al., } \\
1991^{22}\end{array}$ & 1 & 0 & 0 & 0 \\
\hline Spetzler et al., $1992^{11}$ & 2 & $1(50)$ & 0 & 0 \\
\hline King et al., $1993^{23}$ & 4 & 0 & $1(25)$ & 0 \\
\hline $\begin{array}{l}\text { Origitano et al., } \\
1993^{24}\end{array}$ & 2 & 0 & $1(50)$ & 0 \\
\hline Collice et al., $1997^{25}$ & 5 & 0 & $1(20)$ & $1(20)$ \\
\hline Day et al., $1997^{6}$ & 1 & 0 & 0 & 0 \\
\hline Arai et al., $1998^{26}$ & 1 & 0 & $1(100)$ & 0 \\
\hline $\begin{array}{l}\text { Motoyama et al., } \\
2000^{27}\end{array}$ & 1 & 0 & 0 & 0 \\
\hline $\begin{array}{l}\text { Seifert and Stolke, } \\
2003^{28}\end{array}$ & 15 & $6(40)$ & $1(7)$ & $1(7)$ \\
\hline Evans et al., $2004^{29}$ & 1 & 0 & 0 & $1(100)$ \\
\hline Hamel et al., $2005^{30}$ & 1 & $1(100)$ & 0 & 0 \\
\hline $\begin{array}{l}\text { Bambakidis et al., } \\
2009^{31}\end{array}$ & 1 & 1 & 0 & 0 \\
\hline Higa et al., $2009^{32}$ & 1 & 0 & 0 & 0 \\
\hline Present study & 33 & $2(6)$ & $5(16)$ & $5(15)$ \\
\hline
\end{tabular}

are the downside of microsurgical approaches. However, endovascular procedures also carry risks of complications in cases of vertebrobasilar aneurysms. Even with the rapid development of the technology, complications with endovascular approaches, including thromboembolic complications, aneurysm recanalization, intraprocedural hemorrhage, in-stent stenosis, jailed perforators, and formation of pseudoaneurysms, have been reported, and long-term follow-up of the patients is lacking. ${ }^{16,18,19,51-53}$ A recent study on flow-diverting stents for posterior circulation aneurysms ${ }^{54}$ showed a mortality of $12.3 \%$ and

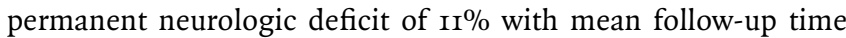
of 9.5 months. For more complex cases, such as fusiform vertebrobasilar aneurysms, the procedural morbidity and mortality after endovascular treatment have been reported at $29 \%$ and $57 \%$, respectively. ${ }^{55}$ The role of microneurosurgery in many vertebrobasilar aneurysm cases is still essential, not only because of the technical means but also because of the reality that many people cannot access high-priced endovascular procedures. To maintain a high-level performance in microneurosurgery, it should be performed in high-volume centers. Therefore, centralization of the service is the best strategy to achieve acceptable outcomes.

\section{CONCLUSIONS}

We have described our experiences of using a modified presigmoid approach to treat vertebrobasilar aneurysms by a single experienced neurosurgeon. The overall results are still acceptable when accounting for the complex location and configuration of the aneurysm. Unfavorable patient outcomes were related to poor preadmission Hunt and Hess grade, aneurysm morphology, and aneurysm size.

\section{ACKNOWLEDGMENTS}

Special thanks to Jörgen Svensson, M.A., from Helsinki University Language Center.

\section{REFERENCES}

I. Grossi PM, Nonaka Y, Watanabe K, Fukushima T. The history of the combined supra- and infratentorial approach to the petroclival region. Neurosurg Focus. 2012;33:E8.

2. Hitselberger WE, House WF. A combined approach to the cerebellopontine angle. A suboccipital-petrosal approach. Arch Otolaryngol. Ig66;84:267-285.

3. Morrison AW, King TT. Experiences with a translabyrinthine-transtentorial approach to the cerebellopontine angle. Technical note. J Neurosurg. I973;38:382-39o.

4. Hakuba A, Nishimura S, Inoue Y. Transpetrosaltranstentorial approach and its application in the therapy of retrochiasmatic craniopharyngiomas. Surg Neurol. I985;24:405-415.

5. Hakuba A, Nishimura S, Jang BJ. A combined retroauricular and preauricular transpetrosaltranstentorial approach to clivus meningiomas. Surg Neurol. I988;30:I08-II6.
6. Day JD, Fukushima T, Giannotta SL. Cranial base approaches to posterior circulation aneurysms. If Neurosurg. I997;87:544-554.

7. Kunihiro N, Goto T, Ishibashi K, Ohata K. Surgical outcomes of the minimum anterior and posterior combined transpetrosal approach for resection of retrochiasmatic craniopharyngiomas with complicated conditions. J Neurosurg. 20I4; I2O:I-II.

8. Lawton MT, Daspit CP, Spetzler RF. Presigmoid approaches to skull base lesions. Adv Tech Stand Neurosurg. I997;23:189-204.

9. Sincoff EH, McMenomey SO, Delashaw JB Jr. Posterior transpetrosal approach: less is more. Neurosurgery. 2007;60(2 suppl I):ONS53-ONS58 [discussion: ONS58-ONS59].

Io. Gross BA, Tavanaiepour D, Du R, Al-Mefty O, Dunn IF. Petrosal approaches to posterior circulation aneurysms. Neurosurg Focus. 2012;33:E9.

II. Spetzler RF, Daspit CP, Pappas CT. The combined supra- and infratentorial approach for lesions of the petrous and clival regions: experience with 46 cases. J Neurosurg. I992;76:588-599.
I2. Gross BA, Tavanaiepour D, Du R, Al-Mefty O, Dunn IF. Evolution of the posterior petrosal approach. Neurosurg Focus. 20I2;33:E7.

I3. Molyneux A, Kerr R, Stratton I, Sandercock P, Clarke M, Shrimpton J, et al. International Subarachnoid Aneurysm Trial (ISAT) of neurosurgica clipping versus endovascular coiling in $2143 \mathrm{pa}-$ tients with ruptured intracranial aneurysms: a randomised trial. Lancet. 2002;360:1267-1274.

I4. Wiebers DO, Whisnant JP, Huston J 3rd, Meissner I, Brown RD Jr, Piepgras DG, et al. Unruptured intracranial aneurysms: natural history, clinical outcome, and risks of surgical and endovascular treatment. Lancet. 2003;362:I03-IIo.

15. Smith GA, Dagostino P, Maltenfort MG, Dumont AS, Ratliff JK. Geographic variation and regional trends in adoption of endovascular techniques for cerebral aneurysms. J Neurosurg. 20II II4:I768-I777.

I6. Wakhloo AK, Mandell J, Gounis MJ, Brooks C, Linfante I, Winer J, et al. Stent-assisted reconstructive endovascular repair of cranial fusiform atherosclerotic and dissecting aneurysms: long- 
term clinical and angiographic follow-up. Stroke. 2008;39:3288-3296.

17. Sekhar LN, Tariq F, Morton RP, Ghodke B, Hallam DK, Barber J, et al. Basilar tip aneurysms: a microsurgical and endovascular contemporary series of Ioo patients. Neurosurgery. 2013;72: 284-298 [discussion: 298-289].

I8. Siddiqui AH, Kan P, Abla AA, Hopkins LN, Levy EI. Complications after treatment with pipeline embolization for giant distal intracranial aneurysms with or without coil embolization. Neurosurgery. 2012;71:E509-E513 [discussion: E513].

19. Pandey AS, Koebbe C, Rosenwasser RH, Veznedaroglu E. Endovascular coil embolization of ruptured and unruptured posterior circulation aneurysms: review of a Io-year experience. Neurosurgery. 2007;60:626-636 [discussion: 636-627].

20. Hunt WE, Hess RM. Surgical risk as related to time of intervention in the repair of intracranial aneurysms. J Neurosurg. I968;28:I4-20.

2I. Lehecka M, Laakso A, Hernesniemi J. Common approaches. In: Lehecka M, Laakso A, Hernesniemi J, eds. Helsinki Microneurosurgery. I. Helsinki, Finland: Druckerei Hohl; 20Iı:I6o-I69.

22. Solomon RA, Smith CR, Raps EC, Young WL, Stone JG, Fink ME. Deep hypothermic circulatory arrest for the management of complex anterior and posterior circulation aneurysms. Neurosurgery. I99I;29:732-737 [discussion 737-738].

23. King WA, Black KL, Martin NA, Canalis RF, Becker DP. The petrosal approach with hearing preservation. J Neurosurg. I993;79:508-514.

24. Origitano TC, Anderson DE, Tarassoli Y, Reichman OH, al-Mefty O. Skull base approaches to complex cerebral aneurysms. Surg Neurol. I993; 40:339-346.

25. Collice M, Arena O, D'Aliberti G, Todaro C, Branca V, Boccardi E, et al. Aneurysms of the vertebro-basilar junction area: preliminary experience in endovascular and surgical management. Acta Neurochir. I997;I39(2):I24-I33.

26. Arai $\mathrm{H}$, Sato K, Miyajima M. Clipping of an aneurysm of a fenestrated basilar artery. $\mathrm{Br} \mathrm{J}$ Neurosurg. I998;I2:I56-I58.

27. Motoyama Y, Ohnishi H, Koshimae N, Kanemoto Y, Kim YJ, Yamada T, et al. Direct clipping of a large basilar trunk aneurysm via the posterior petrosal (extended retrolabyrinthine presigmoid) approach-case report. Neurol Med Chir. 2000;40:632-636.

28. Seifert V, Stolke D. Posterior transpetrosal approach to aneurysms of the basilar trunk and vertebrobasilar junction. J Neurosurg. I996;85: 373-379.

29. Evans JJ, Sekhar LN, Rak R, Stimac D. Bypass grafting and revascularization in the management of posterior circulation aneurysms. Neurosurgery. 2004;55:1036-I049.

3o. Hamel W, Grzyska U, Westphal M, Kehler U. Surgical treatment of a basilar perforator aneurysm not accessible to endovascular treatment. Acta Neurochir. 2005;I47:I283-I286.
3I. Bambakidis NC, Manjila S, Dashti S, Tarr R, Megerian CA. Management of anterior inferior cerebellar artery aneurysms: an illustrative case and review of literature. Neurosurg Focus. 2009;26: E6.

32. Higa T, Ujiie H, Kato K, Kamiyama H, Hori T. Basilar artery trunk saccular aneurysms: morphological characteristics and management. Neurosurg Rev. 2009;32:I8I-I9I [discussion: I9I]

33. Gonzalez LF, Amin-Hanjani S, Bambakidis NC, Spetzler RF. Skull base approaches to the basilar artery. Neurosurg Focus. 2005;I9:E3.

34. Drake CG, Peerless SJ, Hernesniemi JA. Surgery of Vertebrobasilar Aneurysm London, Ontario Experience on 1767 Patients. New York: Springer-Verlag; I996.

35. Randell T, Niemelä M, Kyttä J, Tanskanen P, Määttänen M, Karatas A, et al. Principles of neuroanesthesia in aneurysmal subarachnoid hemorrhage: the Helsinki experience. Surg Neurol. 2006; 66:382-388 [discussion: 388].

36. Nader Sanai AM, Spetzler Robert F. Surgical management of midbasilar and lower basilar aneurysms. In: Quiñones-Hinojosa A, ed. Schmidek. 6th ed. I. Philadelphia, PA: WB Saunders; 2012: 925-936.

37. Abdel Aziz KM. Basilar bifurcation aneurysms: strategies for surgical approach selection. Neuro surgery Quarterly. 2007;I7:IOI-II2.

38. Villavicencio AT, Gray L, Leveque JC, Fukushima T, Kureshi S, Friedman AH. Utility of three-dimensional computed tomographic angiography for assessment of relationships between the vertebrobasilar system and the cranial base. Neurosurgery. 2001;48:318-326 [discussion: 3263I7].

39. Bendok BR, Getch CC, Parkinson R, O'Shaughnessy BA, Batjer HH. Extended lateral transsylvian approach for basilar bifurcation aneurysms. Neurosurgery. 2004;55:174-178 [discussion: 178$]$.

40. Dolenc VV, Skrap M, Sustersic J, Skrbec M, Morina A. A transcavernous-transsellar approach to the basilar tip aneurysms. Br J Neurosurg. I987; I: 25I-259.

4I. Figueiredo EG, Paiva WS, Gomes M, Castro Flores JA, Wen HT, Teixeira MJ. Modified abbreviated transcavernous approach to basilar artery aneurysms-case report. Surg Neurol. 2009;7I: 25-29.

42. Gonzalez LF, Crawford NR, Horgan MA, Deshmukh P, Zabramski JM, Spetzler RF. Working area and angle of attack in three cranial base approaches: pterional, orbitozygomatic, and maxillary extension of the orbitozygomatic approach. Neurosurgery. 2002;50:550-555 [discussion: 555-557].

43. Bendok BR, Rahme RJ, Aoun SG, El Ahmadieh TY, El Tecle NE, Batjer HH, et al. Enhancement of the subtemporal approach by partial posterosuperior petrosectomy with hearing preservation. Neurosurgery. 20I4;IO(suppl 2): I9I-I99 [discussion: I99].
44. Hernesniemi J, Ishii K, Niemela M, Kivipelto L, Fujiki M, Shen H. Subtemporal approach to basilar bifurcation aneurysms: advanced technique and clinical experience. Acta Neurochir Suppl. 2005;94:3I-38.

45. Kawase T, Toya S, Shiobara R, Mine T. Transpetrosal approach for aneurysms of the lower basilar artery. J Neurosurg. I985;63:857-861.

46. McLaughlin N, Martin NA. Extended subtemporal transtentorial approach to the anterior incisural space and upper clival region: experience with posterior circulation aneurysms. Neurosurgery. 2014;Io(suppl I):15-23 [discussion: 23-24].

47. Drake CG. The surgical treatment of aneurysms of the basilar artery. J Neurosurg. I968;29:436-446.

48. Aziz KM, van Loveren HR, Tew JM Jr, Chicoine MR. The Kawase approach to retrosellar and upper clival basilar aneurysms. Neurosurgery. I999;44:I225-I234 [discussion I234-I226].

49. Hafez A, Nader R, Al-Mefty O. Preservation of the superior petrosal sinus during the petrosal approach. J Neurosurg. 20II;II4:I294-I298.

50. Kivisaari RP, Porras M, Ohman J, Siironen J, Ishii K, Hernesniemi J. Routine cerebral angiography after surgery for saccular aneurysms: is it worth it? Neurosurgery. 2004;55:I0I5-I024.

5I. Carneiro A, Rane N, Kuker W, Cellerini M Corkill R, Byrne JV. Volume changes of extremely large and giant intracranial aneurysms after treatment with flow diverter stents. Neuroradiology. 20I4;56:5I-58.

52. Kulcsar Z, Ernemann U, Wetzel SG, Bock A Goericke S, Panagiotopoulos V, et al. High-profile flow diverter (silk) implantation in the basilar artery: efficacy in the treatment of aneurysms and the role of the perforators. Stroke. 20I0;4I: I69o-I696.

53. Nagashima H, Kobayashi S, Tanaka Y, Hongo K. Endovascular therapy versus surgical clipping for basilar artery bifurcation aneurysm: retrospective analysis of II7 cases. J Clin Neurosci. 2004;II: 475-479.

54. Toth G, Bain M, Hussain MS, Moskowitz S, Masaryk T, Rasmussen P, et al. Posterior circulation flow diversion: a single-center experience and literature review. J Neurointerv Surg. 2015;7:574-583.

55. Siddiqui AH, Abla AA, Kan P, Dumont TM Jahshan S, Britz GW, et al. Panacea or problem: flow diverters in the treatment of symptomatic large or giant fusiform vertebrobasilar aneurysms. J Neurosurg. 20I2;II6:1258-I266.

Conflict of interest statement: The authors declare that the article content was composed in the absence of any commercial or financial relationships that could be construed as a potential conflict of interest.

Received 2 April 2016; accepted 2 May 2016

Citation: World Neurosurg. (2016) 92:313-322.

http://dx.doi.org/10.1016/j.wneu.2016.05.001

Journal homepage: www.WORLDNEUROSURGERY.org Available online: www.sciencedirect.com

1878-8750/\$ - see front matter (c) 2016 Elsevier Inc. All rights reserved. 